\title{
COMPLETE STELLAR MODELS FOR MASSIVE STARS
}

\author{
D. SCHAERER \\ Geneva Observatory, CH-1290 Sauverny, Switzerland \\ A. DE KOTER \\ NASA/GSFC USRA, Greenbelt, MD 20771, U.S.A. \\ and \\ W. SCHMUTZ \\ Institut für Astronomie, ETH Zürich, CH-8092 Zürich, Switzerland
}

\begin{abstract}
We present a first step towards complete stellar models for massive stars that treat the entire star including its wind. Our models are based on state-of-the art nonLTE atmospheric and interior modelling. The models yield the stellar evolution under the constraint of realistic atmospheres as well as line-blanketed continuum fluxes and $\mathrm{H}$ and He line profiles. We follow the evolution from OB to LBV, Ofpe/WN, and WR stars. We investigate the effect of line-blanketing during MS evolution. We adress the effect of an the extended atmosphere on the evolution and discuss the first results for WR stars.
\end{abstract}

Key words: stars: Wolf-Rayet - evolution - atmospheres - winds - mass loss

\section{Introduction}

Despite the improvements achieved in both atmospheric and interior modelling of massive stars, comparisons between predicted and observed stellar parameters (review: Maeder \& Conti 1994) remain difficult given the complexity of the physical conditions (non-LTE effects, line-blanketing, and mass loss in winds) in their outer layers. In order to allow sound comparisons, hydrodynamic atmosphere models (Sellmaier et al. 1993; Schaerer \& Schmutz 1994, hereafter SS) are required for spectral analysis and the simplified treatment of the outer layers of evolutionary models must be abandoned particularly for WR stars.

In this work we take a new step towards realistic models of massive stars. Combining line-blanketed non-LTE models for spherically expanding atmospheres and interior models, we construct the first complete stellar models (CoStar), which consistently treat the star from its core to the domain where the wind reaches its terminal velocity. This allows us to study the coupled interior and spectral evolution.

\section{Method and calculations}

A schematic view of our models is shown in Fig. 1. The atmosphere is similar to the hydrodynamic models of SS. The structure can either be obtained from full photosphere-wind models (see SS) or by imposing $\dot{M}$ and a velocity structure. For the calculations presented in this work, we use empirical 


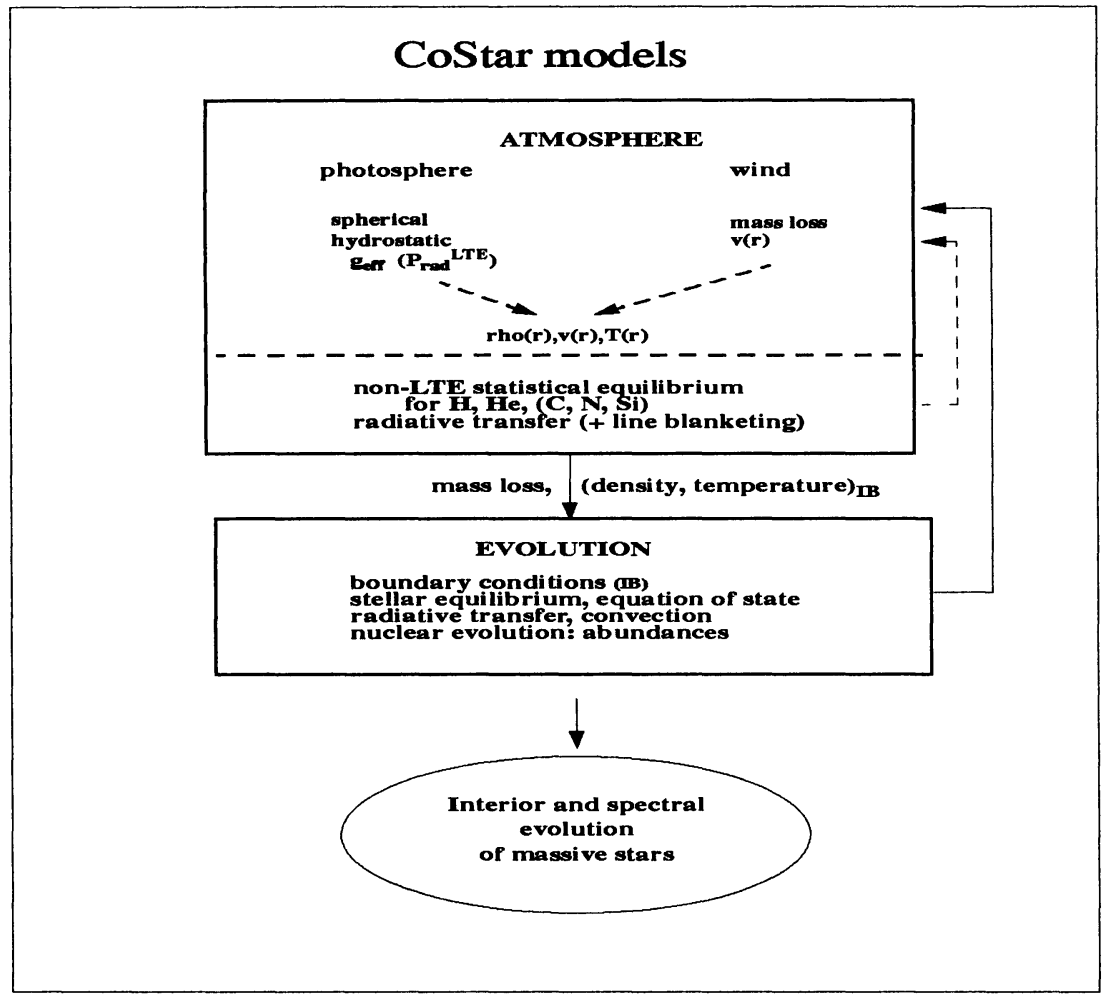

Fig. 1. Overview of the complete stellar models.

$\dot{M}$ values as in Meynet et al. (1993, MMSSC), which are in a good agreement with observations of various galaxies (Maeder \& Meynet 1994). For the temperature structure we either use the method from SS or radiative equilibrium in a spherical grey atmosphere. Here we choose the grey approach and basically determine the $T(r)$ and $\rho(r)$ structure as in de Koter et al. (1994, KLS), with the terminal velocity $v_{\infty}$ from wind models of Leitherer et al. (1992) and $\beta=0.8$ on the MS. The improvement over KLS is that for OB stars we now include full OPAL-opacities at $\tau_{\text {Ross }}>2$ to simultaneously solve for hydrostatic equilibrium and the temperature structure of the photosphere. During the WR phases, due to the high mass-flux of these stars, $\tau_{\star}$ (see below) is usually above the hydrostatic part. For these phases we adopt $\beta=2$.

The non-LTE calculation includes $\mathrm{H}$ and $\mathrm{He}$ and is realised with the ISAWIND code of KLS. In addition, we include line-blanketing (see SS). The transition between the atmosphere and the interior is made at an optical depth $\tau_{\star}=20$, which defines the radius $R_{\star}$ and therefore $T_{\star}$ by virtue of $L=4 \pi \sigma R_{\star}^{2} T_{\star}^{4}$. The values $\dot{M}, \rho$, and $T$ at $\tau_{\star}$ are the outer boundary conditions (IB in Fig. 1) to the interior model, which is modelled with the Geneva stellar evolution code ( $c f$. also Maeder, these proceedings). 

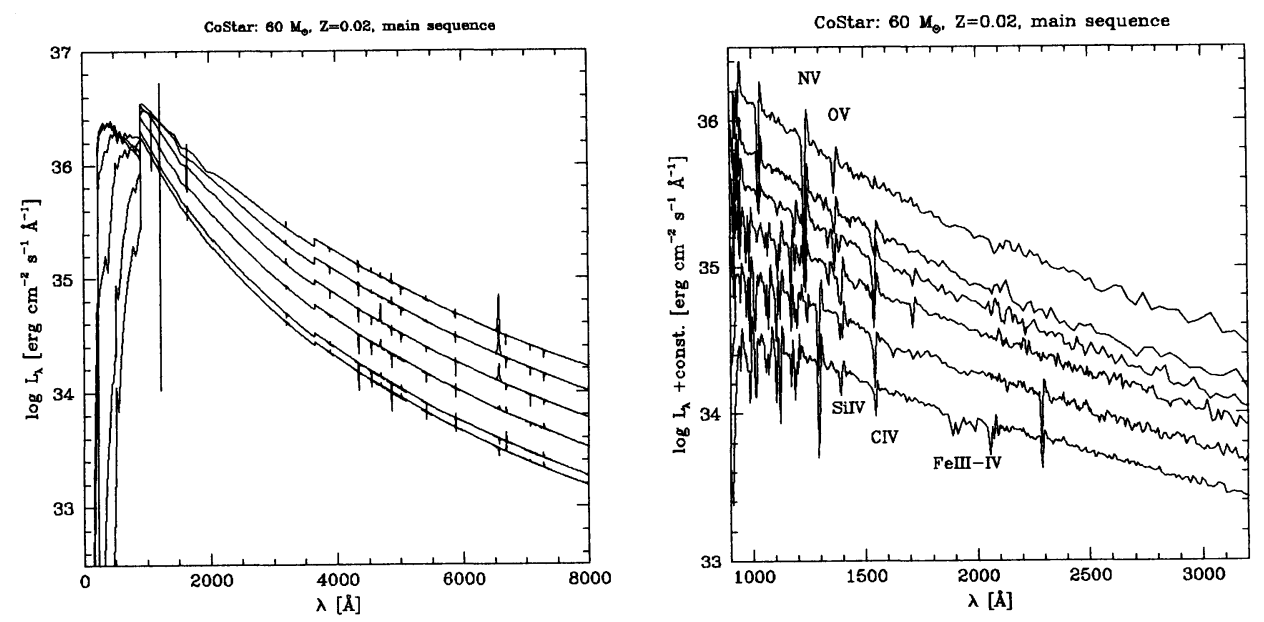

Fig. 2. Left: Theoretical continuum and $\mathrm{H}$ and $\mathrm{He}$ line fluxes along the MS evolution of a $60 \mathrm{M}_{\odot}$ ZAMS star. The models correspond to $\sim$ O5V to B5I. Right: Predicted UV fluxes from Monte Carlo simulations (see SS) along the main sequence shown from top to bottom. The most important spectral features are labeled.

\section{Results: consistent spectral and interior evolution}

In Fig. 2 (left), we show the resulting blanketed continuum fluxes with the most important $\mathrm{H}$ and $\mathrm{He}$ lines along the MS evolution of a $60 \mathrm{M}_{\odot}$ star. Note the increasing effect of blanketing on the observable UV continuum during the evolution towards the Terminal Age Main Sequence (TAMS). The right panel shows the predicted UV fluxes including up to $\sim 65000$ lines. These clearly reveals the effects of blanketing at $\lambda<1300 \AA$ and around $2000 \AA$.

Fig. 3 shows the HR-diagram for the $85 \mathrm{M}_{\odot}$ track. Note that the MS evolution of the CoStar model proceeds at slightly higher $T_{\text {eff }}$ than the standard model. The explanation is the following (see Fig. 3). Given the very proximity of the Eddington limit (cf. Lamers \& Fitzpatrick 1988) of these models, we calculate the photospheric structure with $g_{\text {eff }}$ taking only electron scattering into account. To reach a given optical depth this therefore requires a slightly higher density than in the plane parallel case, which results in a larger external pressure and therefore smaller radii. As an indirect consequence, $\dot{M}$ decreases with increasing $T_{\text {eff }}$, which results in a slightly overluminous evolution.

Note that this procedure for the photosphere is adopted since the use of Rosseland opacities to calculate $g_{\text {eff }}$ would otherwise lead to density inversions, which are probably not real in the presence of a wind. An improved treatment would therefore require both a complete hydrodynamic solution including line and continuum forces and a correct treatment of possible density inversions ( $c f$. Maeder 1992), which is beyond the scope of the present 

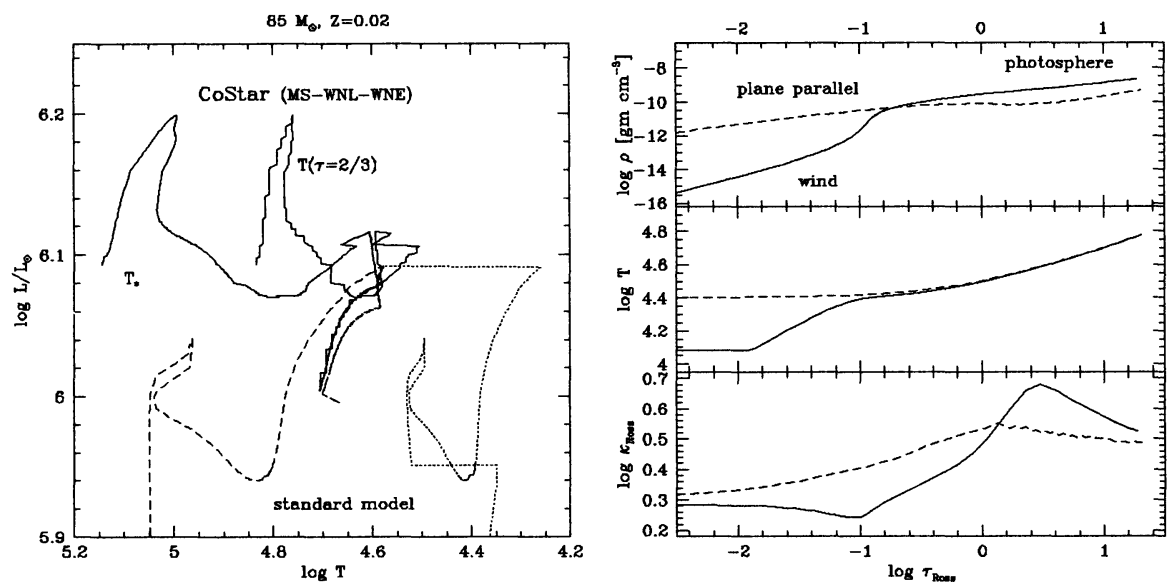

Fig. 3. Left: Comparison of the standard evolutionary track from MMSSC (dotted: wind correction according to SSMM, dashed: no correction) with the CoStar model (full) for a $85 \mathrm{M}_{\odot}$ model. Note the large temperature difference between $T_{2 / 3}$ and $T_{\star}$. Right: Run of the opacities, and density and temperature structures of a plane parallel and extended atmosphere close to the TAMS.

work. From this it is clear that the importance of the effect shown above on the MS evolution might be slightly overestimated.

Once the WR phase is reached, the atmospheric extension becomes sufficiently important that the effective temperature of CoStar models differs from that from models with hydrostatic atmospheres. This is illustrated by the difference between $T_{\star}$ and $T\left(\tau_{\text {Ross }}=2 / 3\right)$ (see Fig. 3), which in our case is of the order of 0.2 dex without and $<0.3$ dex including line-blanketing for the phases calculated in these models. Previously, this effect was estimated by applying a simple temperature correction formula (Schaller et al. 1992, SSMM). These first results indicate an overestimation of the role of the lines in the temperature correction of SSMM for the hot WN stars. More detailed studies for cooler WR models and WC stars are in progress.

\section{References}

Lamers, H.J.G.L.M., Fitzpatrick, E.L. 1988, $A p J$ 324, 279

de Koter, A., Schmutz, W., Lamers, H.J.G.L.M. 1993, $A 8 A$ 277, 561 (KLS)

Leitherer, C., Robert, C., Drissen, L. 1992, ApJ 401, 596

Maeder, A. 1992, in: C. de Jager \& H. Nieuwenhuijzen (eds.), Instabilities in Evolved Super- and Hypergiants (Amsterdam: North Holland), p. 138

Maeder, A., Conti, P. 1994, Ann. Rev. $A \& A$ in press

Maeder, A., Meynet, G., $1994 A \mathscr{E} A$ in press (MM)

Meynet, G., Maeder, A., Schaller, G., Schaerer, D., Charbonnel, C. 1993, A\&A Suppl. 103, 97 (MMSSC)

Schaerer, D., Schmutz, W. 1994, $A \& A$ in press (SS)

Schaller, G., Schaerer, D., Meynet, G., Maeder, A. 1992, A\&A Suppl. 96, 269 (SSMM)

Sellmaier, F., Puls, J., Kudritzki, R.P., Gabler, A., Gabler, R., Voels, S.A. 1993, $A \mathfrak{E} A$ 273, 533 


\section{DISCUSSION:}

Langer: In the case of WR mass loss I would expect, from my models, to have already large outflow velocities at the point where you fit the wind solution to the hydrostatic core. What is your assumption on the velocity at this point?

Schaerer: Indeed, we know that for the WR stars with strong mass loss the continuum is formed at regions with supersonic outflow velocities. Regrettably we do not have a theory yet which is capable to describe the trans-sonic velocity field and the mass loss rates for WR's.

For the present calculations we therefore have a discontinuity for the velocity at the lower boundary, since we assume hydrostatic equilibrium below the atmosphere.

I would nevertheless like to point out that for a typical WR star at this depth one precisely is in a zone where there is a very large opacity peak (due to Fe mainly), which can lead to local supra Eddington luminosities (as is well known in cooler supergiants). Theoretically it remains unclear whether this leads to density invessions related to a convective zone, Rayleigh-Taylor instabilities or if this would be able to start the optically thick WR wind.

Polcaro: Is there in your models any indications of a discontinuity in the mass loss rate, corresponding to the LBV phase?

Schaerer: We have not yet explored the full hydrodynamic behaviour of the envelope and the winds of LBVs. Possible answers can probably be found in a combination of Pauldrach \& Puls (1990, A\&A 237, 409) for the winds and Stothers \& Chiu (1993, ApJ 408, L85) or Glatzel et al. for the envelope.

Shaviv: How do you fit the moving wind with the hydrostatic star, at constant $M, \tau$ or what? Schaerer: The boundary between the atmosphere and the stellar interior is defined at a Rosseland optical depth (typically 20) where radiation at all wavelengths is thermalized and LTE is attained. For OB stars this point is always localized in the subsonic region, in the hydrostatic part of the photosphere.

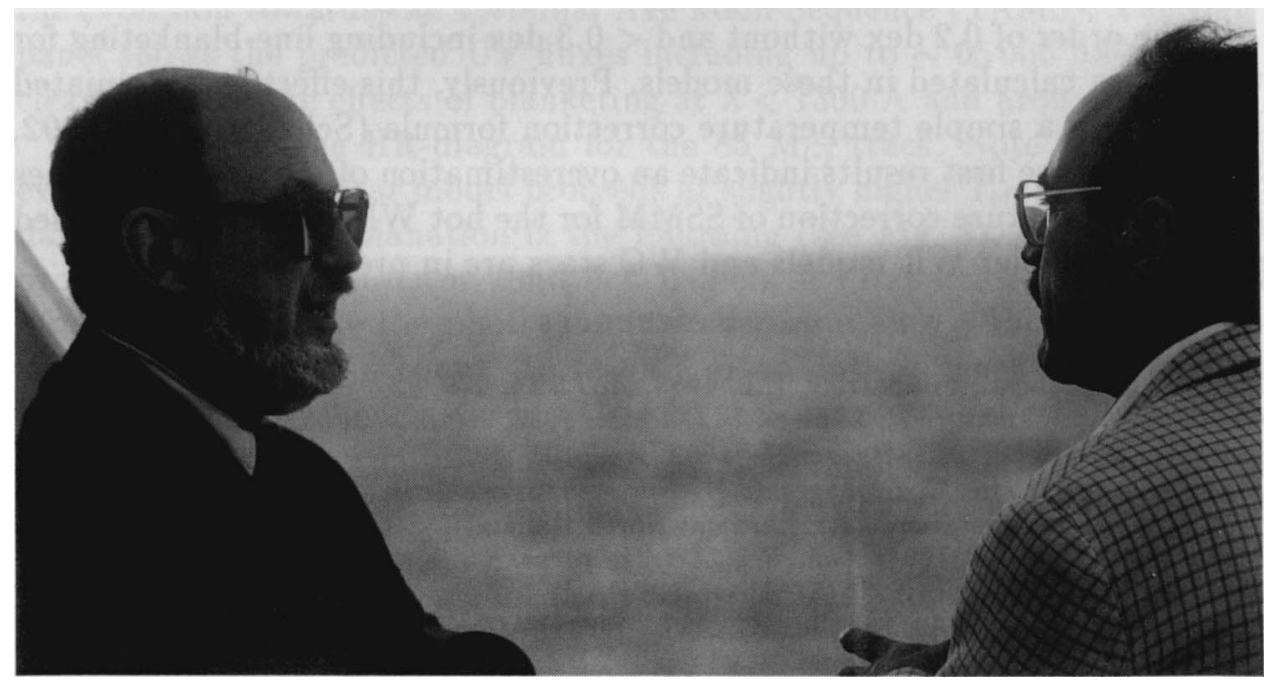

Conti, Maeder 